\title{
Statistical properties of Klauder-Perelomov coherent states for the Morse potential
}

\author{
M. Daoud ${ }^{1, *}$ and D. Popov ${ }^{2}$ \\ ${ }^{1}$ Max Planck Institute for the Physics of Complex Systems \\ Dresden, Germany \\ ${ }^{2}$ University "Politehnica" of Timişoara, Department of Physics \\ Timişoara, Romania
}

November 6, 2018

\begin{abstract}
We present in this letter a realistic construction of the coherent states for the Morse potential using the Klauder-Perelomov approach . We discuss the statistical properties of these states, by deducing the Q- and P-distribution functions. The thermal expectations for the quantum canonical ideal gas of the Morse oscillators are also calculated.
\end{abstract}

${ }^{*}$ Permanent adress: LPMC, Faculty of Sciences, University Ibn Zohr, Agadir, Morocco 


\section{Introduction}

The one-dimensional Morse potential was first introduced as a useful model for diatomic molecules in 1929 [1]. Since its introduction, the Morse oscillator has proved very useful for various problems in diverse fields of physics and chemistry (diatomic and polyatomic molecular systems, quantum chemistry, spectroscopy, chemical bonds) $[2,3,4,5,6,7]$ (and the references therein).

On the other hand, since the pioneer papers of Glauber, Klauder and Perelomov about the coherent states of the one-dimensional harmonic oscillator [8], [9], and their different applications in physics [10], this approach has been paid attention also for other potentials. The three way to define the coherent states for the onedimensional harmonic oscillator (i.e.: 1. as states which minimize the uncertainty relations; 2. as states which diagonalize the lowering operator and 3 . as states obtained by action of the displacement operator on the bound state), which in this case are convergent (lead to the same results), for an another anharmonic potential lead to the three kind of states, generically named: Barut-Girardello, KlauderPerelomov and Gazeau-Klauder [11], [12], [13].

Based on these papers, the coherent states was constructed for a series of potentials (see, [14], [15], [16], [17], [18]).

For the pseudoharmonic oscillator the Barut-Girardello coherent states, as well as the photon-added Barut-Girardello coherent states was constructed [19], [20].(see also, [21]).

For the Morse oscillator, the construction of generalized coherent states was begin by the papers of [3]. Recently, Dong have constructed the coherent states based on the SU(2) realization for the Morse potential [22], Fakhri and Chenaghlou have constructed the Barut-Girardello coherent states for the Morse potential [23], while Roy and Roy, and, also, Popov have constructed and examined the properties of the Gazeau-Klauder coherent states for the same potential [24], [25]. So, the examination of the Klauder-Perelomov coherent states for the Morse potential, which is the main aim of this paper becomes a naturally task.

This paper is organized as follows. In Sec. 2, after a brief recall of the main facts and properties of the Morse oscillator, we deduce the corresponding raising and lowering operators and we construct the Klauder-Perelomov coherent states for this oscillator. Section 3 is devoted to the examination of the statistical properties of these coherent states, by examining a quantum canonical ideal gas of Morse oscillators, in thermodynamical equilibrium with the reservoir. the concluding remarks will be given in Sec. 4 .

\section{Klauder-Perelomov coherent states}

We shall begin by recalling some basic facts of the Morse potential, mainely the eigenstates and corresponding creation and annihilation operators. The hamiltonian

for a one dimensional quantum particle evolving in the Morse potential is given by 


$$
H_{-}^{l}=-\frac{d^{2}}{d^{2} x}+V^{l}(x)
$$

where

$$
V^{l}(x)=(l+1)^{2}-(2 l+3) e^{-x}+e^{-2 x}
$$

with $l$ is an integer to simplify. The energies are given by

$$
E_{n}^{l}=(l+1)^{2}-(l+1-n)^{2}
$$

where the quantum number $n$ takes the finite sequence of values: $0,1, \ldots, l$.

The discrete spectrum of the Morse potential is finite and this fact is very important in the construction of his coherent states as we will discuss in the sequel of this note.

Because the ground state is zero energy, it is well known that one can factorize the Hamiltonian as

$$
H_{-}^{l}=X_{l}^{+} X_{l}^{-}
$$

where the operators $X_{l}^{ \pm}$(hermitian conjugated of each other,i.e $\left(X_{l}^{+}\right)^{\dagger}=X_{l}^{-}$) are given by

$$
X_{l}^{ \pm}=\mp \frac{d}{d x}-\left(e^{-x}-(l+1)\right) .
$$

From the supersymmetric quantum mechanics, it is well established that the supersymmetric partners

$$
H_{\mp}^{l}=X_{l}^{ \pm} X_{l}^{\mp}=-\frac{d^{2}}{d x^{2}}+e^{-2 x}-(2(l+1) \pm 1) e^{-x}+(l+1)^{2}
$$

have the same energy spectra, but different eigenstates. Indeed, it is easy to verify that

$$
H_{+}^{l}=H_{-}^{l-1}+(2 l+1)
$$

which implies that the eigenstates of $H_{+}^{l}$ can be obtained from $\left|\psi_{n}^{l}\right\rangle$ corresponding to $H_{-}^{l}$ by substituting $l$ by $l-1$. The Schrödinger equations for $H_{\mp}^{l}$ are

$$
H_{\mp}^{l}\left|\psi_{n}^{l-\frac{1}{2} \pm s}\right\rangle=(l+1)^{2}-\left(l+\frac{1}{2} \pm s-n\right)^{2}\left|\psi_{n}^{l-\frac{1}{2} \pm s}\right\rangle,
$$

where $s=\frac{1}{2}$. It is straightforward to check, for each $l$, the intervening relations

$$
X_{l}^{\mp} H_{\mp}^{l}=H_{ \pm}^{l} X_{l}^{\mp}
$$

Due to the latter relations the operators $X_{l}^{ \pm}$link the Hilbert spaces $\mathcal{H}^{l \pm s-\frac{1}{2}}$ and $\mathcal{H}^{l \mp s-\frac{1}{2}}$ spanned by the bounded states of the Morse potential. They connect eigenfunctions with the same energy. Indeed one has

$$
\begin{gathered}
X_{-}^{l}\left|\psi_{n}^{l}\right\rangle=\sqrt{E_{n}^{l}} e^{i \alpha(2(l-n)+1)}\left|\psi_{n-1}^{l-1}\right\rangle \\
X_{+}^{l}\left|\psi_{n}^{l-1}\right\rangle=\sqrt{E_{n+1}^{l}} e^{-i \alpha(2(l-n)+1)}\left|\psi_{n+1}^{l}\right\rangle
\end{gathered}
$$


where $\alpha \in \mathbf{R}$. The operators $X_{-}^{l}$ and $X_{+}^{l}$ are not the ladder operators of the Hamiltonian $H_{-}^{l}$. To define the creation and annihilation operators for $H_{-}^{l}$, we define the operator

$$
U=\sum_{m}\left|\psi_{m}^{l}\right\rangle\left\langle\psi_{m}^{l-1}\right|
$$

satisfying $U^{+} U=1$ and $\left|\psi_{n-1}^{l}\right\rangle=U\left|\psi_{n-1}^{l-1}\right\rangle$. Using the transformation $U$, define a new pair of operators

$$
A_{+}^{l}=X_{+}^{l} U^{+} \quad, \quad A_{-}^{l}=U X_{-}^{l}
$$

Combining (10), (11) and (12), we get the actions of $A_{+}^{l}$ and $A_{-}^{l}$ on the eigenstates $\left\{\left|\psi_{n}^{l}\right\rangle\right\}$ as

$$
\begin{gathered}
A_{-}^{l}\left|\psi_{n}^{l}\right\rangle=\sqrt{E_{n}^{l}} e^{i \alpha(2(l-n)+1)}\left|\psi_{n-1}^{l}\right\rangle \\
A_{+}^{l}\left|\psi_{n}\right\rangle=\sqrt{E_{n+1}^{l}} e^{-i \alpha(2(l-n)+1)}\left|\psi_{n+1}^{l}\right\rangle
\end{gathered}
$$

The set of operators $\left\{A_{-}^{l}, A_{+}^{l}\right\}$ are the creation and annihilation operators of $H^{l}=A_{+}^{l} A_{-}^{l}=X_{+}^{l} X_{-}^{l}$. They satisfy the following commutation relations

$$
\left[A_{-}^{l}, A_{+}^{l}\right]=-2 N+(2 l+1)
$$

where the operator $N$ is given by

$$
N\left|\psi_{n}^{l}\right\rangle=n\left|\psi_{n}^{l}\right\rangle .
$$

The algebra generated by $A_{-}^{l}, A_{+}^{l}$ and $N$ provides the appropriate tool to build up the coherent states for the Morse potential.

The so-called Klauder-Perelomov coherent states for quantum system embedded in the Morse potential are defined

$$
|z, \alpha\rangle=\exp \left(z A_{+}^{l}-\bar{z} A_{-}^{l}\right)\left|\psi_{0}^{l}\right\rangle
$$

Expanding the displacement operator and using the actions of the creation and annihilation operators, it comes

$$
|z, \alpha\rangle=\sum_{n=0}^{l} z^{n} I_{n}^{l}(|z|) e^{-i \alpha E_{n}}\left|\psi_{n}^{l}\right\rangle
$$

where

$$
I_{n}^{l}(|z|)=\sum_{j=0}^{\infty} \frac{\left(-|z|^{2}\right)^{j}}{(n+2 j) !} \Delta^{l}(n+1, j)
$$

The quantities $\Delta$ which occur in the last formula are give by

$$
\Delta^{l}(n+1, j)=\frac{n !(2 l+1) !}{(2 l+1-n) !} \sum_{i_{1}=1}^{n+1} E_{i_{1}}^{l} \sum_{i_{2}=1}^{i_{1}+1} E_{i_{2}}^{l} \ldots \sum_{i_{j}=1}^{i_{j-1}+1} E_{i_{j}}^{l}
$$

with $\Delta^{l}(n+1,0)=\frac{n !(2 l+1) !}{(2 l+1-n) !}$. They satisfy the following reccurence equation

$$
\Delta^{l}(n+1, j)=\sqrt{2 n(l+1)-n^{2}} \Delta^{l}(n, j)+\sqrt{2 n l+2 l+1-n^{2}} \Delta^{l}(n+2, j-1) .
$$


Setting

$$
J_{n}^{l}(|z|)=|z|^{n} \sqrt{\frac{(2 l+1-n) !}{n !(2 l+1) !}} I_{n}^{l}(|z|),
$$

we get the first order differential equation

$$
\frac{d J_{n}^{l}(|z|)}{d|z|}=J_{n-1}^{l}(|z|)-\left(2 n l+2 l+1-n^{2}\right) J_{n+1}^{l}(|z|) .
$$

The solution of this equation takes the simple form

$$
J_{n}^{l}(|z|)=\frac{1}{n !}(\cos (|z|))^{l-1}(\operatorname{tg}(|z|))^{n}
$$

and the Morse coherent states rewrite as

$$
|Z, \alpha\rangle=\left(1+|Z|^{2}\right)^{-\frac{l}{2}} \sum_{n=0}^{l} \sqrt{\frac{l !}{n !(l-n) !}} Z^{n} e^{-i \alpha E_{n}^{l}}\left|\psi_{n}^{l}\right\rangle
$$

where $Z=\frac{z}{|z|} t g(|z|)$. They have the property of strong continuity in the label space and completeness in the sense that there exists a positive measure such that they solve the resolution to identity. The appropriate form of this resolution is

$$
\int d \mu(Z, \bar{Z})|Z, \alpha\rangle\left\langle Z, \alpha\left|=\sum_{n=0}^{l}\right| \psi_{n}^{l}\right\rangle\left\langle\psi_{n}^{l}\right| .
$$

To determine the measure $d \mu(Z, \bar{Z})$, we assume his isotropy and we set

$$
d \mu(Z, \bar{Z})=\left(1+|Z|^{2}\right)^{l} h\left(|Z|^{2}\right)|Z| d|Z| d \theta / \pi
$$

with $Z=|Z| e^{i \theta}$. Substituting Eq. (28) into Eq.(27), we obtain the following sum which should be satisfied by the function $\left.h\left(x=|Z|^{2}\right)\right)$

$$
\int_{0}^{\infty} x^{n} h(x) d x=\frac{n !(l-n) !}{l !} .
$$

The inverse Mellin transform gives

$$
h(x)=\frac{l+1}{(1+x)^{l+2}} .
$$

This result can be obtained also by using the definition of Meijer's G-function and the Mellin inversion theorem [26].

Due to phase introduced in the definitions of annihilation and creation operators, the obtained coherent states are temporally stable. Indeed

$$
e^{-i t H_{-}^{l}}|Z, \alpha\rangle=|Z, \alpha+t\rangle
$$

The physical utility of Klauder-Perelomov coherent sates for the Morse potential (26) in different applications consists in the calculation of the expectation (mean) 
values of a certain physical observable $A$ which characterizes the quantum system embedded in the Morse potential, with respect to $\mid Z, \alpha>$ :

$$
\begin{gathered}
<Z, \alpha|A| Z, \alpha>\equiv<A>_{Z}= \\
=\left(1+|Z|^{2}\right)^{-l} \sum_{n, m=0}^{l} \sqrt{\left(\begin{array}{c}
l \\
n
\end{array}\right)\left(\begin{array}{c}
l \\
m
\end{array}\right)} Z^{n} \bar{Z}^{m} e^{-\mathrm{i} \alpha\left(\mathrm{E}_{\mathrm{n}}^{\mathrm{l}}-\mathrm{E}_{\mathrm{m}}^{1}\right)}<\psi_{m}^{l}|A| \psi_{n}^{l}>
\end{gathered}
$$

where we have used the binomial coefficients [27]:

$$
\left(\begin{array}{l}
l \\
n
\end{array}\right)=\frac{l !}{n !(l-n) !}=\frac{\Gamma(l+1)}{\Gamma(n+1) \Gamma(l+1-n)} .
$$

From a practical point of view, the most important operators are the diagonal operators in the eigenstates-basis $\mid \psi_{n}^{l}>$ corresponding to $H_{-}^{l}$, so that

$$
<\psi_{m}^{l}|A| \psi_{n}^{l}>=a_{n} \delta_{m n}
$$

If $A=N^{s}$, where the operator $N$ is given by Eq. (17) and $s$ is an integer, we have:

$$
<A>_{Z}=\frac{1}{(1+x)^{l}} \sum_{n=0}^{l}\left(\begin{array}{l}
l \\
n
\end{array}\right) n^{s} x^{n}=\frac{1}{(1+x)^{l}}\left(x \frac{d}{d x}\right)^{s}(1+x)^{l},
$$

where we have used the simplified notation: $x=|Z|^{2}$.

For $s=1$ and 2 we obtain, successively:

$$
\begin{gathered}
<N>_{Z}=l \frac{x}{1+x} \\
<N^{2}>_{Z}=l \frac{x}{1+x}+l(l-1)\left(\frac{x}{1+x}\right)^{2} .
\end{gathered}
$$

These expectation values are useful in order to calculate the second-order correlation function for the morse oscillator [28]:

$$
\left(g^{2}\right)_{Z}=\frac{<N^{2}>_{Z}-<N>_{Z}}{\left(<N>_{Z}\right)^{2}}=\frac{l-1}{l}<1 .
$$

Moreover, the Mandel Q-parameter is [29]

$$
Q_{Z}=<N>_{Z}\left[\left(g^{2}\right)_{Z}-1\right]=-\frac{x}{1+x}<0 .
$$

These quantities provide information about the inherent statistical properties of the Klauder-Perelomov coherent states of the Morse oscillator $\mid Z, \alpha>$. These properties depend on the analytical expressions of the functions (39) and (40) as depending on the variable $x=|Z|^{2}$. Because of the structure of these functions, $\left(g^{2}\right)_{Z}$ and $Q_{Z}$ can be evaluated analytically. Generally speaking, the states $|Z, \alpha\rangle$ exhibit sub-Poissonian statistics for those values of $x=|Z|^{2}$ for which $Q_{Z}<0$ (or 
$\left(g^{2}\right)_{Z}<1$ )(antibunching effect), Poisson statistics for values for which $Q_{Z}=0$ (or $\left(g^{2}\right)_{Z}=1$ ) and supra-Poissonian statistics for values of $Z$ for which $Q_{Z}>0$ (or $\left(g^{2}\right)_{Z}>1$ ) (bunching effect). As we see from the above equations, the KlauderPerelomov coherent states of the Morse oscillator $\mid Z, \alpha>$ are sub-Poissonian, for all values of the variable $|Z|^{2}$.

By putting $A=H_{-}^{l}$ and using the eigenvalue equation (3), we obtain the action identity:

$$
\begin{gathered}
<H_{-}^{l}>_{Z}=\frac{1}{(1+x)^{l}} \sum_{n=0}^{l}\left(\begin{array}{l}
l \\
n
\end{array}\right) x^{n} E_{n}^{l}= \\
=2(l+1)<N>_{Z}-<N^{2}>_{Z}=l(2 l+1) \frac{x}{1+x}-l(l-1)\left(\frac{x}{1+x}\right)^{2} \equiv f(x) .
\end{gathered}
$$

We see that this is a certain function of the label $x=|Z|^{2}$ and this fact characterizes all the coherent states corresponding to systems with a finite dimensional energy spectrum, as it was mentioned earlier [24], [25].

\section{Statistical properties}

We consider a quantum gas of the Morse oscillators in thermodynamic equilibrium with the reservoir (the thermostat) at temperature $T$, which obeys the quantum canonical distribution. Also, we consider that the individual Morse oscillators are in such states which are labelled by number state vectors $\left|\psi_{n}^{l}\right\rangle$. The corresponding normalized density operator for a fixed $l$-parameter (or, equivalently, for a fixed Morse oscillator) is then

$$
\rho_{l}=\frac{1}{Z_{l}} \sum_{n=0}^{l} e^{-\beta \varepsilon_{n}^{l}}\left|\psi_{n}^{l}><\psi_{n}^{l}\right|,
$$

where $\beta=\left(k_{B} T\right)^{-1}, k_{B}$ is Boltzmann's constant and $Z_{l}$ - the normalization constant, i.e. the partition function for a certain fixed parameter $l$ :

$$
Z_{l}=\sum_{n=0}^{l} e^{-\beta \varepsilon_{n}^{l}}
$$

In the above exponential it appear the dimensional Morse eigenenergies:

$$
\varepsilon_{n}^{l} \equiv \frac{\hbar \omega}{2(l+1)} E_{n}^{l}=\hbar \omega n-\frac{\hbar \omega}{2(l+1)} n^{2}
$$

where $\omega$ is the angular frequency for the Morse oscillator with a fixed parameter $l$.

The Q-function (the Husimi's function), i.e. the diagonal elements of the density operator in the representation of coherent states, is

$$
<Z, \alpha\left|\rho_{l}\right| Z, \alpha>=\frac{1}{Z_{l}} \sum_{n=0}^{l} e^{-\beta \varepsilon_{n}^{l}}\left(\begin{array}{l}
l \\
n
\end{array}\right) x^{n} .
$$


It is not difficult to verify that the normalization condition of the density operator is accomplished:

$$
\operatorname{Tr} \rho_{l}=\int d \mu(Z, \bar{Z})<Z, \alpha\left|\rho_{l}\right| Z, \alpha>=1
$$

where we have used the following integral [27], [30]:

$$
\int_{0}^{\infty} d x x^{\alpha-1}(x+z)^{-\gamma}=z^{\alpha-\gamma} B(\alpha, \gamma-\alpha)=z^{\alpha-\gamma} \frac{\Gamma(\alpha) \Gamma(\gamma-\alpha)}{\Gamma(\gamma)} .
$$

If we consider the dimensional Morse Hamiltonian, and the dimensional eigenvalues of energy, instead of $H_{-}^{l}$ and $E_{n}^{l}$, then the corresponding energy exponential can be written as follows:

$$
\beta \varepsilon_{n}^{l}=\beta \hbar \omega n-\beta \frac{\hbar \omega}{2(l+1)} n^{2} \equiv \mathcal{A} n-\mathcal{B} n^{2} .
$$

For most of the diatomic molecules $\mathcal{B} \ll \mathcal{A}$. So, the limits of the parameter $2(l+1)$ are very large, e.g. 37.1586 for $\mathrm{H}_{2}$ molecule, i.e. for a "light" molecule and 348.78 for $\mathrm{I}_{2}$, a "heavy" molecule [31]. As a consequence, the quantity $\mathcal{B}$ can be regarded as a perturbation constant and the energy exponential can be expanded in the power series as follows:

$$
e^{-\beta \varepsilon_{n}^{l}}=e^{-\mathcal{A} n} \sum_{k=0}^{\infty} \frac{\mathcal{B}^{k}}{k !} n^{2 k}=\sum_{k=0}^{\infty} \frac{\mathcal{B}^{k}}{k !}\left(\frac{d}{d \mathcal{A}}\right)^{2 k}\left[e^{-\mathcal{A}}\right]^{n} .
$$

We can also use the following operator identity:

$$
\sum_{k=0}^{\infty} \frac{\mathcal{B}^{k}}{k !}\left(\frac{d}{d \mathcal{A}}\right)^{2 k} \equiv \exp \left[\mathcal{B}\left(\frac{d}{d \mathcal{A}}\right)^{2}\right]
$$

Taking into account this ansatz, after the straightforward calculations, we can write the Q-function in the following manner:

$$
<Z, \alpha\left|\rho_{l}\right| Z, \alpha>=\frac{1}{Z_{l}} \exp \left[\mathcal{B}\left(\frac{d}{d \mathcal{A}}\right)^{2}\right]\left(\frac{1+x e^{-\mathcal{A}}}{1+x}\right)^{l} .
$$

Because the Klauder-Perelomov coherent states for the Morse oscillator $|Z, \alpha\rangle$ form an overcomplete set of states, they may be used as a basis set despite the fact that they are not orthogonal. Let us perform the diagonal expansion of the density operator $\rho_{l}$ in the coherent states basis:

$$
\rho_{l}=\frac{1}{Z_{l}} \int d \mu(Z, \bar{Z})\left|Z, \alpha>P_{l}\left(|Z|^{2}\right)<Z, \alpha\right| .
$$

In order to find the quasi-probability distribution function $P_{l}\left(|Z|^{2}\right)$ (or the $\mathrm{P}$ function) from the above diagonal expansion, we observe that the equation

$$
<f\left|\rho_{l}\right| g>=\frac{1}{Z_{l}} \int d \mu(Z, \bar{Z})<f\left|Z, \alpha>P_{l}\left(|Z|^{2}\right)<Z, \alpha\right| g>
$$


must be fulfilled for any arbitrary vectors $\langle f|$ and $|g\rangle$ from the Hilbert space (or, for any vectors from the basis $\mid Z, \alpha>$ or $\left.\left|\psi_{n}^{l}\right\rangle\right)$.

The left-hand side of this equation is

$$
L H S=\frac{1}{Z_{l}} \sum_{n=0}^{l} e^{-\beta \varepsilon_{n}^{l}}<f\left|\psi_{n}^{l}><\psi_{n}^{l}\right| g>
$$

while, after the angular integration

$$
\frac{1}{2 \pi} \int_{-\pi}^{\pi} d \theta e^{-\mathrm{i}(n-m) \theta}=\delta_{n m}
$$

the right-hand side becomes

$R H S=\frac{1}{Z_{l}} \sum_{n=0}^{l}\left(\begin{array}{l}l \\ n\end{array}\right)<f\left|\psi_{n}^{l}><\psi_{n}^{l}\right| g>2(l+1) \int_{0}^{\infty} d|Z||Z|^{2 n+1} \frac{1}{\left(1+|Z|^{2}\right)^{l+2}} P_{l}\left(|Z|^{2}\right)$.

After the variable change $x=|Z|^{2}$ we must have:

$$
\int_{0}^{\infty} d x x^{n} \frac{1}{(1+x)^{l+2}} P_{l}(x)=e^{-\beta \varepsilon_{n}^{l}} \frac{1}{l+1}\left(\begin{array}{l}
l \\
n
\end{array}\right)^{-1}=e^{-\beta \varepsilon_{n}^{l}} \frac{1}{l+1} \frac{\Gamma(n+1) \Gamma(l+1-n)}{\Gamma(l+1)}
$$

where $P_{l}(x)$ is an unknown function. In order to determine it, we extend the previous ansatz referring to the energy exponential [25].

It is obvious that the $\mathrm{P}$-function also depends on the quantities $\mathcal{A}$ and $\mathcal{B}$, besides the variable $x$. This leads to the idea that $P_{l}(x) \equiv P_{l}(x, \mathcal{A}, \mathcal{B})$ can also be expanded in a power series similarly to the energy exponential (49) in the following manner:

$$
P_{l}(x)=\sum_{k=0}^{\infty} \frac{\mathcal{B}^{k}}{k !}\left[\left(\frac{d}{d \mathcal{A}}\right)^{k} P_{l}(x, \mathcal{A}, \mathcal{B})\right]_{\mathcal{B}=0} \equiv \sum_{k=0}^{\infty} \frac{\mathcal{B}^{k}}{k !}\left(\frac{d}{d \mathcal{A}}\right)^{2 k} X_{l}(x, \mathcal{A}),
$$

where the function $X_{l}(x, \mathcal{A})$ is to be determined.

By inserting Eqs. (49) and (58) into the equation (57), performing the function change obtain:

$$
X_{l}(x, \mathcal{A})=\frac{1}{\Gamma(l+2)}(1+x)^{l+2} h_{l}(x, \mathcal{A})
$$

as well as by extending the natural values of $n$ to complex $s$ such as $n=s-1$, we get to the following Stieltjes moment problem:

$$
\int_{0}^{\infty} d x x^{s-1} h_{l}(x, \mathcal{A})=e^{\mathcal{A}} \frac{1}{\left(e^{\mathcal{A}}\right)^{s}} \Gamma(s) \Gamma(l+2-s) .
$$

The solution of such a problem is [26] 


$$
h_{l}(x, \mathcal{A})=e^{\mathcal{A}} \Gamma(l+2) \frac{1}{\left(1+e^{\mathcal{A}} x\right)^{l+2}} .
$$

Finally, the P-function is

$$
P_{l}(x)=\exp \left[\mathcal{B}\left(\frac{d}{d \mathcal{A}}\right)^{2}\right]\left[e^{\mathcal{A}}\left(\frac{1+x}{1+e^{\mathcal{A}} x}\right)^{l+2}\right] .
$$

where we have use the operator identity (50).

The scalar product of two Klauder-Perelomov coherent states for the Morse oscillator is

$$
<Z, \alpha \mid Z^{\prime}, \alpha>=\frac{\left(1+\bar{Z} Z^{\prime}\right)^{l}}{\left(1+|Z|^{2}\right)^{\frac{l}{2}}\left(1+\left|Z^{\prime}\right|^{2}\right)^{\frac{l}{2}}} .
$$

From the trace condition $\left(\operatorname{Tr} \rho_{l}=1\right)$ applied to the diagonal expansion (52) and the scalar product, it is not difficult to prove that the P-function satisfies the normalization condition:

$$
\frac{1}{Z_{l}} \int d \mu(Z, \bar{Z}) P_{l}\left(|Z|^{2}\right)=1
$$

Let us verify this equation using the our obtained expression for the P-function (62). Thus, we have

$$
\begin{array}{r}
I \equiv \frac{1}{Z_{l}}(l+1) \int_{0}^{2 \pi} \frac{d \theta}{\pi} \frac{1}{2} \int_{0}^{\infty} d x \frac{1}{(1+x)^{2}} \exp \left[\mathcal{B}\left(\frac{d}{d \mathcal{A}}\right)^{2}\right]\left[e^{\mathcal{A}}\left(\frac{1+x}{1+e^{\mathcal{A}} x}\right)^{l+2}\right]= \\
=\frac{1}{Z_{l}}(l+1) \exp \left[\mathcal{B}\left(\frac{d}{d \mathcal{A}}\right)^{2}\right]\left[e^{\mathcal{A}} \int_{0}^{\infty} d x(1+x)^{l}(1+\exp \mathcal{A} x)^{-l-2}\right] .
\end{array}
$$

The last integral is of the following kind [27], [30]:

$$
\int_{0}^{\infty} \frac{(a x+b)^{\beta-1}}{(c x+d)^{\beta+1}}=\frac{(a d)^{\beta}-(b c)^{\beta}}{\beta(a d-b c)(c d)^{\beta}}, \quad[c d>0 ; \quad a d \neq b c ; \quad \operatorname{Re} \beta \geq 1]
$$

This lead to the results:

$$
\begin{aligned}
I=\frac{1}{Z_{l}} \exp \left[\mathcal{B}\left(\frac{d}{d \mathcal{A}}\right)^{2}\right]\left[\frac{1-\left(e^{\mathcal{A}}\right)^{l+1}}{\left(1-e^{\mathcal{A}}\right)\left(e^{\mathcal{A}}\right)^{l}}\right]= \\
=\frac{1}{Z_{l}} \exp \left[\mathcal{B}\left(\frac{d}{d \mathcal{A}}\right)^{2}\right]\left[\sum_{n=0}^{l}\left(e^{-\mathcal{A}}\right)^{n}\right]=1 .
\end{aligned}
$$

So, our obtained expression for the P-function (62) is correct. 
In this way, the diagonal representation of the normalized density operator of the Morse oscillator in the Klauder-Perelomov coherent states representation is

$$
\rho_{l}=\frac{1}{Z_{l}} \int d \mu(Z, \bar{Z}) \exp \left[\mathcal{B}\left(\frac{d}{d \mathcal{A}}\right)^{2}\right]\left[e^{\mathcal{A}}\left(\frac{1+|Z|^{2}}{1+e^{\mathcal{A}}|Z|^{2}}\right)\right]|Z, \alpha><Z, \alpha| .
$$

Then the thermal expectation value (the thermal average) of an observable $A$ concerning the Morse oscillator is given by

$$
<A>_{l}=\operatorname{Tr}\left(\rho_{l} A\right)=\frac{1}{Z_{l}} \int d \mu(Z, \bar{Z}) P_{l}\left(|Z|^{2}\right)<Z, \alpha|A| Z, \alpha>.
$$

If the operator $A$ is an integer power $s$ of the number operator $N$, then, using Eqs. (69), (36, (47), as well as (49), we obtain the expected result:

$$
<N^{s}>_{l}=\frac{1}{Z_{l}} \sum_{n=0}^{l} n^{s} e^{-\beta \varepsilon_{n}^{l}}=\frac{1}{Z_{l}}(-1)^{s}\left(\frac{\partial}{\partial \mathcal{A}}\right)^{s} Z_{l} .
$$

With this result we can define and calculate the thermal second-order correlation function $\left(g^{(2)}\right)$ and the thermal Mandel parameter $Q_{l}$, i.e. the thermal analogue of the corresponding functions for the Klauder-Perelomov coherent states $|Z, \alpha\rangle$ (see, Eqs. (39) and (40)):

$$
\begin{gathered}
\left(g^{(2)}\right)_{l}=\frac{<N^{2}>_{l}-<N>_{l}}{\left(<N>_{l}\right)^{2}}=1+\frac{1}{\frac{\partial}{\partial \mathcal{A}} \ln Z_{l}}+\frac{\left(\frac{\partial}{\partial \mathcal{A}}\right)^{2} \ln Z_{l}}{\left(\frac{\partial}{\partial \mathcal{A}} \ln Z_{l}\right)^{2}}, \\
Q_{l}=<N>_{l}\left[\left(g^{(2)}\right)_{l}-1\right]=-1-\frac{\left(\frac{\partial}{\partial \mathcal{A}}\right)^{2} \ln Z_{l}}{\frac{\partial}{\partial \mathcal{A}} \ln Z_{l}} .
\end{gathered}
$$

The difference between these results and the similar results for the GazeauKlauder quasi-coherent states of the Morse oscillator [25] (i.e. the absence of the term $1 / 2$ ) is due definition of the Morse energy eigenstates (3), comparing by $E_{n}=$ $\hbar \omega(n+1 / 2)-\frac{\hbar \omega}{2(l+1)(n+1 / 2)^{2}}$ (see, Eq. (3) in Ref. [25] ).

\section{Discussion and outlook}

In conclusion, we have given a construction of Klauder-Perelomov coherent states associated with the bound states of the Morse potential.These states possess all properties of the usual coherent states as continuity, overcompletion, non-orthogonality and temporal stability, being defined on the entire complex space of the variable $Z$.

In order to examine the statistical properties of these states, we have concentrated our attention on a quantum system which consists on the quantum canonical ideal gas of Morse oscillators, in thermodynamic equilibrium with the reservoir (thermostat). Firstly, we have obtained the expressions of the diagonal elements 
of the density operator in the Klauder-Perelomov coherent states (the Husimi's Qfunction), as well as the diagonal representation of the density operator and the corresponding P-function. With these functions we are able to express the thermal expectations (thermal averages) for the physical quantities which characterize the Morse oscillator quantum system. The second-order correlation function and the Mandel parameter, as well as their corresponding thermal pairs, offer the information on the character of the coherent states, versus the Poisson distribution function.

Besides the implicit construction of the Klauder-Perelomov coherent states for the Morse potential and examination of their properties, the main results of this paper are the derivation of the integration measure and the deduction of the expressions for Q- and P-functions and, consequently, the thermal averages calculated with these functions. In our opinion, the above obtained results seems to be entire new, because, to our knowledge, these results have not yet been published in the literature.

By using the general expression for thermal averages one can easy be deduced some interesting concrete thermal averages for the Morse oscillators quantum canonical ideal gas as: free and internal energy, entropy and molar heat capacity at the constant volume and so on.

Acknowledgements: M.D is grateful to Max Planck Institute for the Physics of Complex systems for its support . 


\section{References}

[1] P. M. Morse, Phys. Rev. 34 (1929) 57.

[2] L. Pauling and E. B. Wilson Jr. - Introduction to Quantum Mechanics, McGrawHill Co., New York, 1935.

[3] M. M. Nieto and L. M. Simmons Jr. - Phys. Rev. A 19, 2, 438 (1979).

[4] M. L. Sage - Chem. Phys. 35, 3, 375 (1978).

[5] V. S. Vasan and R. J. Cross - J. Chem. Phys. 78, 6, 3869 (1983).

[6] J. P. Dahl and M. Sprinborg - J. Chem. Phys. 88, 7, 4335 (1988).

[7] D. Popov - Description of the Multielectronical Systems by means of the Density Matrix Method, Ph. D. Thesis work, University of Craiova, Romania (in Romanian, unpublished), 1986.

[8] J.R.Klauder and B.S Skagerstam, Coherent states-Applications in Physics and Mathematical Physics (World Scientific,Singapore,1985).

[9] A.Perelomov, Generalized Coherent States and their Applications,Texts and Monographs in Physics, (Spinger-Verlag,1986).

[10] S.T.Ali, J-P. Antoine and J-P.Gazeau, Coherent States, Wavelets and their generalizations( Springer-Verlag, New York,2000).

[11] J.R.Klauder, J.Phys.A:Math.Gen. 29 (1996) L 293.

[12] J-P.Gazeau and J-R.Klauder, J.Phys.A:Math.Gen. 32 (1999) 123.

[13] J-P. Antoine, J-P.Gazeau, P.M.Monceau, J.R.Klauder and K.A.Penson J.Math.Phy 42 (2001) 2349.

[14] M. Daoud and V. Hussin, J.Phys.A:Math.Gen. 35 (2002) 7381.

[15] A.H. El Kinani and M. Daoud, J.Phys.A:Math.Gen.34 (2001) 5373.

[16] A.H. El Kinani and M. Daoud, Phys. Lett.A 283 (2001)291.

[17] A.H. El Kinani and M. Daoud, Inter. Jour.Mod. Phys.B 15 (2001)2465.

[18] A.H. El Kinani and M. Daoud, J.Math.Phys. 35 (2001) 2297.

[19] D. Popov, J.Phys.A:Math.Gen. 34 (2001) 5283.

[20] D. Popov, J.Phys.A:Math.Gen. 35 (2002) 7205.

[21] M. Daoud and E. H. El Kinani, J.Phys.A:Math.Gen. 35 (2002) 2639. 
[22] S. H. Dong, R. Lemus, A. Frank, Int. J. Quant. Chem. 86 (2002) 433; S. H. Dong, Can. J. Phys. 80 (2002) 129.

[23] H. Fakhri, A.Chenaghlou, Phys. Lett. A 310 (2003) 1.

[24] B. Roy and P. Roy, Phys.Lett.A 296 (2002) 187.

[25] D. Popov, Phys. Lett. A 316 (2003) 369.

[26] A. M. Mathai, R. K. Saxena, Lecture Notes in Mathematics, Vol. 348: Generalized Hypergeometric Functions with Applications in Statistics and Physical Sciences, Springer, Berlin-Heidelberg-New York, 1973.

[27] I. S. Gradshteyn and I. M. Ryzhik, Table of Integrals, Series and Products, Academic Press, New York,1980.

[28] D. F. Walls, G. J. Milburn, Quantum Optics, Springer, Berlin, 1994.

[29] A. I. Solomon, Phys. Lett. A 196 (1994) 29.

[30] A. P. Proudnikov, Yu. A. Brychkov, O. I. Marichev, Integrals and Series, Elementarly Functions, Nauka, Moscow, 1981 (in Russian).

[31] D. Popov, Physica Scripta 63 (2001) 257. 